\title{
Characteristics of flow over two tandem cylinders of different diameters
}

\author{
Yong Tao Wang \\ Suzhou University of Science and Technology, P. R. China \\ email : 2216732391@qq.com
}

\begin{abstract}
Keywords: Navier-Stokes equations; Tandem cylinders; Lift coefficients; Pressure distribution; Finite element method
\end{abstract}

\begin{abstract}
Two-dimensional flow over two circular cylinders of different diameters in tandem arrangement at low Reynolds numbers has been numerically investigated in this study. The diameter ratio between the upstream control cylinder and the downstream one was varied from 0.1 to 1.0. The studied Reynolds number based on the diameter of the downstream main cylinder were 100 and 150. The gap between the control cylinder and the main cylinder ranged from 0.1 to 4.0 times the diameter of the main cylinder. Mutual interference between the two cylinders at close proximity, however, caused significant change in parameters of the hydrodynamic characteristics, such as fluctuating lift forces, fluctuating pressure distributions, when the spacing between the cylinders was changed. The changes in these hydrodynamic characteristics were systematically analyzed in this study. It is concluded that the gap ratio and the diameter ratio between the two cylinders have important effects on the lift coefficients and pressure distribution.
\end{abstract}

\section{Introduction}

Most structures on land and in the ocean are in multiple forms and are confronted by a fluid flow. Vibrations of these structures due to fluid flows reduce the life of the respective installations and must therefore be taken into account in the design of the structure. For assessment of this vibration, it is important to understand the interaction of multiple structures in a flow. A tandem arrangement of two circular cylinders is a basic example of an array of multiple structures. The common use of the cylindrical-shaped body in various fields of engineering stimulated the investigation on flow around two circular cylinders in a tandem arrangement.

Previous investigations of tandem configurations by Bearrman and Wadcock(1973), Zdravkovich (1977,1987), Igarashi (1981), Williamson (1985), Sumner et al.(2000), Alam et al. (2008), Sharman et al. (2005), Zdravkovich (1987), Zhao et al.(2005,2007)have revealed considerable complexity in fluid dynamics as the spacing between the cylinders is changed.

In this paper, the flow past two circular cylinders of different diameters is investigated numerically. The aim of this study is to investigate the effects of the two cylinders of different diameters in the tandem arrangement on lift coefficients and pressure distribution. The two-dimensional field Navier-Stokes equations are solved by using a finite volume method. While the downstream main cylinder diameter $D$, is fixed, the upstream control cylinder diameter $d$, is varied from $0.1 D$ to $D$. The Reynolds number based on the diameter of the downstream cylinder is 100 and 150. The gap $G$ between the cylinders ranges from 0.1 to 4.0 times the diameter of the downstream cylinder $D$. The effects of the gap ratio $G / D$ and the diameter ratio $d / D$ between the two cylinders on lift coefficients and pressure distribution around the cylinders and flow characteristics are investigated. 


\section{Governing equations and numerical method}

The two tandem cylinders considered in this study are shown in Figure 1. The position of the upstream cylinder can be uniquely determined by the gap ratio $G / D$ and the diameter ratio $d / D$ between the two cylinders. The flow is in the positive direction of the x-axis, $\theta$ is an angle in the counterclockwise direction, starting from the positive direction of the $\mathrm{x}$-axis, as shown in Figure 1.

The two-dimensional Navier-Stokes equations together with the continuity equation are solved by using a finite volume method. The temporal integration used in the present study is of second-order accuracy. The lift coefficients $\left(C_{\mathrm{L}}\right)$ on the cylinder are calculated

$$
\text { as } C_{L}=\frac{F_{L}}{0.5 \rho U^{2} D}=-\int_{0}^{2 \pi} \frac{p}{\rho U^{2}} \sin \theta d \theta+\frac{v}{U^{2}} \int_{0}^{2 \pi}\left(\frac{\partial v}{\partial x}-\frac{\partial u}{\partial y}\right) \cos \theta d \theta
$$

Where $F_{\mathrm{L}}$ are the total lift forces, $U$ is the free-stream velocity, $u, v$ is velocity components in the $x$-and $y$-direction, respectively. $p$ is fluid pressure, $D$ is a characteristic dimension of the cylinder.

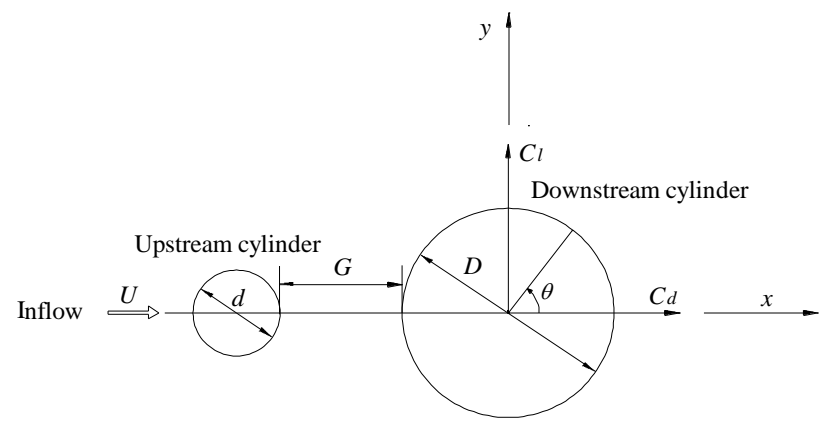

Figure. 1 Definition figure

\section{Lift coefficients acting on two tandem cylinders}

The maximum amplitude value and the root mean square value of lift coefficients, $C_{\mathrm{L}}$ and $C_{\mathrm{Lrms}}$ on the main cylinder for $R e=100$ and 150 are shown in Figure 2 and figure 3 as functions of the locations of the control cylinders, respectively. The variations of $C_{\mathrm{L}}$ and $C_{\mathrm{Lrms}}$ on the main cylinder with the different gap spacing are similar at the same Reynolds number, and the corresponding lift coefficients increase slightly with $R e$ changing from 100 to 150 . Note that magnitudes of $C_{\mathrm{L}}$ and $C_{\mathrm{Lrms}}$ remain significantly small ( smaller than the lift coefficients of isolated cylinder ) in the region $0.1<G / D<2.5$. However, in the region between $G / D=2.5$ and 4.0 , the magnitudes of $C_{\mathrm{L}}$ and $C_{\mathrm{Lrms}}$ experience sudden jumps, and the more $d / D$ is large, the more $C_{\mathrm{L}}$ and $C_{\mathrm{Lrms}}$ are large. The maximum increase in the lift coefficients on the main cylinder is about 5 times of the single cylinder. The shedding vortex street is the main source leading to the fluctuating lift force on the main cylinder. Furthermore, the magnitudes of the fluctuating surface pressure, induced by the shedding vortex street, strongly depend on the strength and vortex formation length of the shedding vortex.

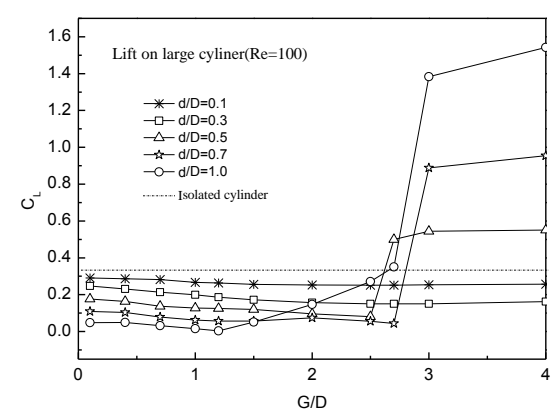

(a) $R e=100$

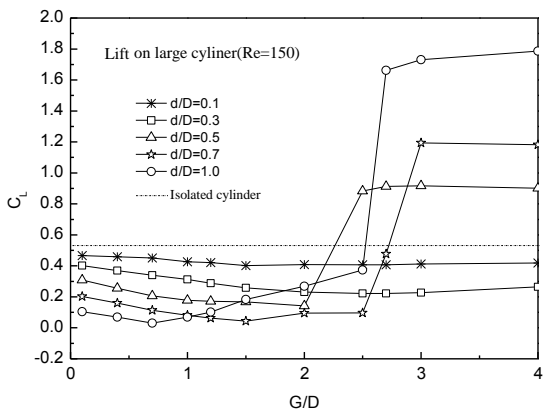

(b) $R e=150$

Figure. 2 The maximum amplitude value of lift coefficients on the downstream cylinder 


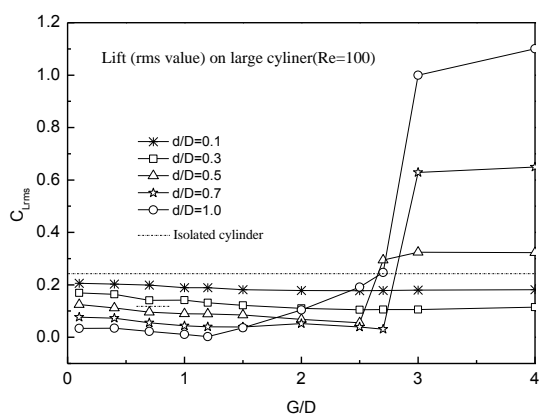

(a) $R e=100$

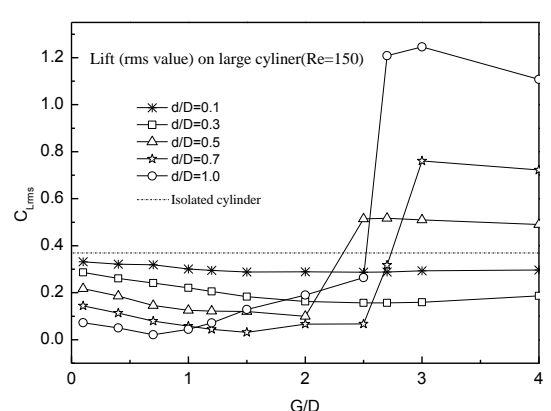

(b) $\operatorname{Re}=150$

Figure. 3 The root mean square value of lift coefficients on the downstream cylinder

\section{Pressure distribution on two tandem cylinders}

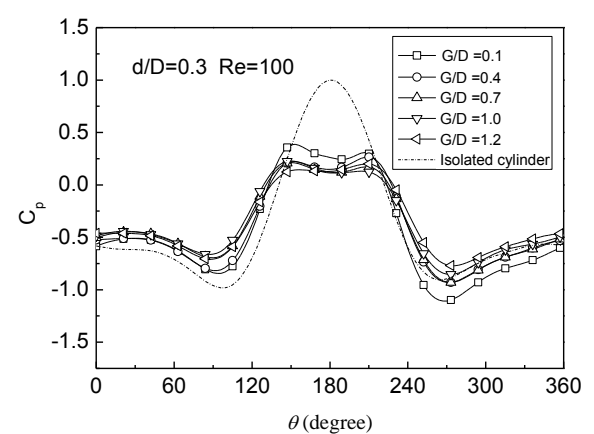

(a) $G / D=0.1-1.2$

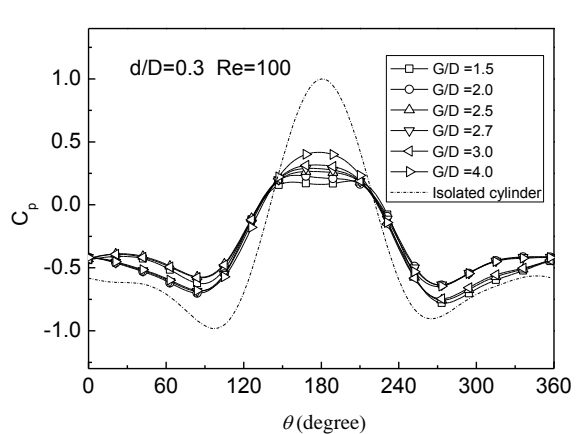

(b) $G / D=1.5-4.0$

Figure. 4 Time-averaged pressure coefficient distribution along the surface of the downstream cylinder.

Figure. 4 shows the distribution of pressure coefficient $C_{\mathrm{p}}$, along the surface of the downstream cylinder for various spacings with $d / D=0.3$ at $R e=100$, including the results for a single cylinder. $C_{\mathrm{p}}$ is defined as

$$
C_{p}=\frac{2\left(p-p_{0}\right)}{\rho U^{2}}
$$

Where $p$ is the static pressure, and $p_{0}$ is the pressure at the faraway location main cylinders. The magnitude of pressure becomes zero at $\theta=141^{\circ}$ and $\theta=219^{\circ}$ and becomes maximum positive at $\theta=180^{\circ}$ (the stagnation point), and becomes maximum negative at $\theta=96^{\circ}$ and $\theta=264^{\circ}$ for the single cylinder. One can notice that an control cylinder significantly reduces $C_{\mathrm{p}}$ at the front stagnation point $\left(\theta=180^{\circ}\right)$, and increases $C_{p}$ at the base suction point $\left(\theta=0^{\circ}\right)$. Therefore, the effective drag reduction can be obtained if the control cylinder is placed at the optimum $G / d$ locations. For $G / D<2.0$, a peak on the front side of the main cylinder is apparent in each of the pressure distributions. It represents the reattachment of the shear layer from the upstream cylinder. These features of pressure distribution are similar to that in previous studies of Prasad.

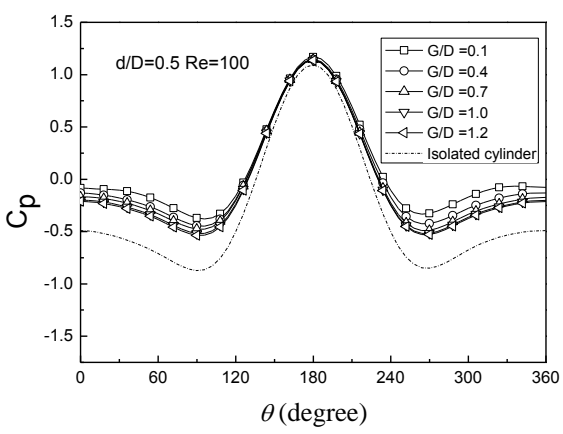

(a) $G / D=0.1-1.2$

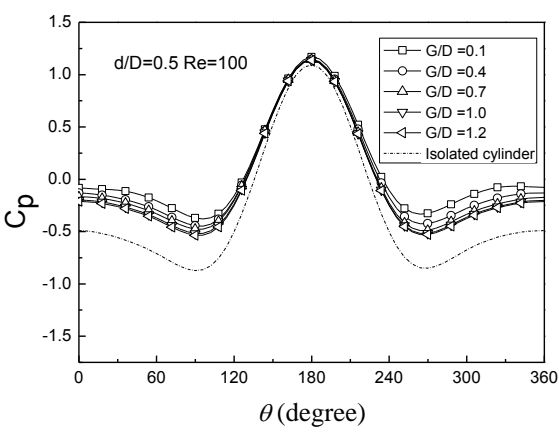

(b) $G / D=1.5-4.0$

Figure. 5 Time-averaged pressure coefficient distribution along the surface of the upstream cylinder. 
Figure 5 shows the distribution of pressure coefficient $C_{p}$ along the surface of the upstream cylinder for various spacings with $d / D=0.5$ at $R e=100$. One can notice that $C_{p}$ of the control cylinder stays nearly constant at the stagnation point $\left(\theta=180^{\circ}\right)$, and increases significantly at the base suction point $\left(\theta=0^{\circ}\right)$. The more $G / D$ is small, the more $C_{p}$ is large in the back region of the upstream cylinder. $C_{\mathrm{p}}$ on the front of the control cylinder is not almost affected by the downstream cylinder.

\section{Conclusions}

The numerical investigations of the effects of the gap ratio and the diameter ratio on the flow past two tandem circular cylinders at $R e=100$ and 150 are carried out. The instantaneous flow patterns and other quantitative information are obtained, which helps a better understanding of the complex flow characteristics around the cylinders in the tandem arrangement. The major results can be summarized as follows:

In general, in the range of $G / D<2.5$, the existence of the control cylinder causes that the mean lift coefficients are smaller than that of a single cylinder, and the lift coefficients decrease with increasing $d / D$. For the small control cylinder diameter $(d / D=0.1,0.3)$, the lift coefficients stay nearly constant, and approach that of a single cylinder in all $G / D$. Then, for big control cylinder diameter $(d / D=0.5,0.7,1.0)$, those steeply increase with increasing $G / D$ in the range of $2.5<G / D$ $<4.0$, and the more $d / D$ is large, the more $C_{\mathrm{L}}$ are large. In any case, $C_{\mathrm{L}}$ and $C_{\mathrm{Lrms}}$ are larger extremely than that of the isolated cylinder.

The upstream cylinder substantially reduces the pressure of the main cylinder in the vicinity of the front of stagnation point with a secondary effect of increasing the base suction pressure. Obviously, the larger the control cylinder is, the larger magnitude of drag reduction we may anticipate. $C_{\mathrm{p}}$ of the upstream control cylinder stays nearly constant at the stagnation point and increases abviously at the base suction point.

\section{References}

[1] Bearman, P. W., and Wadcock, A. J. 1973. The interaction between a pair of circular cylinder normal to a stream. Journal of Fluid Mechanics, 61, 499-511.

[2] Zdravkovich, M. M. 1977. Review of flow interference between two circular cylinders in various arrangements. ASME. Journal of Fluids Engineering, 99, 618-633.

[3] Zdravkovich, M. M. 1987. The effect of interference between circular cylinders in cross flow. Journal of Fluids and Structure, 1, 239-261.

[4] Igarashi, T. 1981. Characteristics of the flow around two circular cylinders arranged in tandem (1st report). Bulletin of the Japan Society of Mechanical Engineering, 24, 323-331.

[5] Williamson, C. H. K. 1985. Evolution of a single wake behind a pair of bluff bodies. Journal of Fluid Mechanics, 159, 1-18.

[6] Sumner, D., Price, S. J., and Paidoussis, M. P. 2000. Flow-pattern identification for two staggered circulation cylinders in cross-flow. Journal of Fluid Mechanics, 411, 263-303.

[7] Alam, M., and Zhou. Y. 2008. Strouhal numbers, forces and flow structures around two tandem cylinders of different diameters. Journal of Fluids and Structure, 24, 505-526.

[8] Sharman, B., Lien, F. S., Davidson, L., and Norberg. C. 2005. Numerical predictions of low Reynolds number flows over two tandem circular cylinders. International Journal for Numerical Methods in Fluids, 47, 423-447.

[9] Zdravkovich, M. M. 1987. The effect of interference between circular cylinders in cross flow. 
Journal of Fluids and Structure, 1, 239-261.

[10] Zhao, M., Cheng. L., Teng, B., and Liang, D. F. 2005. Numerical simulation of viscous flow past two circular cylinders of different diameters. Applied Ocean Research, 27, 39-55.

[11] Zhao, M., Cheng, L., Teng, B., and Dong, G. 2007. Hydrodynamic forces on dual cylinders of different diameters in steady currents. Journal of Fluids and Structure, 23, 59-83.

[12] Prasad, A. and Williamsion, C. H. K. 1997. A method for the reduction of bluff body drag. Journal of Wind Engineering and Industrial Aerodynamics, 69, 155-167. 